\title{
О прогнозе скорости корональных выбросов массы по радионаблюдениям
}

\author{
Ю.T. Ццап ${ }^{1,2}$, Е.А. Исаева ${ }^{3}$ \\ 1 ФГБУН “Крымская астрофизическая обсерватория РАН”, Научный, Крым, 298409 \\ yur@craocrimea.ru \\ 2 Главная (Пулковская) астрономическая обсерватория РАН, Санкт-Петербург, Россия \\ 3 Радиоастрономический институт НАН Украины, Харьков, Украина \\ isaevaode@gmail.com
}

Поступила в редакцию 16 ноября 2017 г.

\begin{abstract}
Аннотация. Исходя из анализа данных Radio Solar Telescope Network для 124 солнечных протонных событий, было установлено, что коэффициент корреляции $r$ между скоростью корональных выбросов массы $V_{C M E}$ и интегральным потоком микроволнового излучения $\int F d t$ составляет около 0.8 . Обнаружена заметная корреляция $(r \approx 0.66)$ между скоростью роста микроволнового излучения и темпом замедления дрейфа радиовсплесков II типа в диапазоне 25-180 МГц. Полученные результаты предполагают, что генерация ударных волн происходит в области вспышечного энерговыделения, и микроволновые наблюдения могут быть использованы для прогноза скоростей $V_{C M E}$.

ON THE PREDICTION OF THE CORONAL MASS EJECTION BASED ON RADIO OBSERVATIONS, by Yu.T. Tsap, E.A. Isaeva. In terms of the analysis of the Radio Solar Telescope Network data obtained for 124 solar proton events it has been established that the correlation coefficient $r$ between the coronal mass ejection velocities $V_{C M E}$ and integral fluxes of microwave emission $\int F d t$ is about 0.8 . A relatively good correlation $(r \approx 0.66)$ was found between the growth rate of microwave emission and the deceleration of frequency drift in the range of $25-180 \mathrm{GHz}$. The obtained results suggest that the shock wave generation occurs in the region of flare energy release and microwave observations can be used to predict $V_{C M E}$.
\end{abstract}

Ключевые слова: Солнце, КВМ, ударные волны, радиоизлучение

\section{1 Введение}

Для изучения корональных выбросов массы (KBM) используют изображения, полученные на коронографах, поэтому KВМ хорошо видны только в плоскости неба. Вследствие этого измерение скоростей KBM $V_{C M E}$, распространяющихся в направлении Земли, становится проблематичным. Между тем анализ микроволнового магнитотормозного излучения солнечных вспышек может быть использован для прогноза значений $V_{C M E}$. Кроме того, не следует также исключать наличие тесной связи между скоростями ударных волн, на которых, как считается, происходит ускорение солнечных космических лучей, и КВМ. Именно эти вопросы и являются предметом настоящих исследований. 


\section{2 Основные критерии протонности событий и исходные данные}

Используемая нами выборка включала 124 протонных события, зарегистрированных с 06.11.1997 по 20.09.2015 гг. с энергией протонов > 1 МэВ, которые одновременно сопровождались континуальными микроволновыми радиовсплесками ( $\mu$-всплески) IV типа и KBM. Из них 76 событий присутствуют в каталоге протонных событий Мирового центра данных ${ }^{1}$, а 48 были отождествлены нами самостоятельно на основе критериев, разработанных в НИРФИ РАН. Это объясняется тем, что на сайте приводится список протонных событий лишь с энергией протонов $>10$ МэВ, а накладывающиеся события не всегда разделяются.

Среди наиболее важных критериев, на основе которых проводилось отождествление потоков протонов со вспышками на диске Солнца, отметим следующие:

1. Наличие U- и W-образного типа частотного радиоспектра континуальных радиовсплесков IV типа в диапазоне 25-15400 МГц. Такие типы частотного радиоспектра имеют глубокий минимум в дециметровом диапазоне и четко выраженные максимумы в сантиметровом и метровом.

2. Мощная метровая-декаметровая компонента континуальных радиовсплесков IV типа, что свидетельствует о благоприятных условиях выхода протонов (Дайбог и др., 1987).

3. Большая эффективная длительность микроволновых всплесков на частоте спектрального микроволнового максимума, что свидетельствует об обогащении солнечных космических лучей протонами. В частности, в работе (Дайбог и др., 1989) показано, что для ускорения субрелятивистских протонов требуется значительно больше времени, чем для электронов.

4. Зависимость времени распространения протонов, их спектра и темпа увеличения потоков от гелиографической долготы протонной вспышки (Очелков, 1986).

Для отождествления протонных событий со вспышками мы использовали оригинальные записи рентгеновского излучения Солнца и интенсивности потока протонов по данным Geostationary Operational Environmental Satellites (GOES), а также результаты спектральных наблюдений радиоизлучения Солнца в диапазоне 25-15400 МГц (Radio Solar Telescope Network - RSTN). Kроме того, при проведении анализа нами привлекались следующие каталоги:

- оригинальные записи интенсивности потока протонов с энергией $>1$ МэВ и мощности рентгеновского излучения в диапазонах $0.05-0.4$ и $0.1-0.8$ нм $^{2}$;

- оригинальные записи динамических спектров в диапазоне 25-180 МГц ${ }^{3}$;

- оригинальные записи радиоизлучения Солнца на частотах 245, 410, 610, 1415, 2695, 4995, 8800, 15400 МГц ${ }^{4}$;

- табличные данные скорости ударных волн в диапазоне 25-180 МГц ${ }^{5}$;

- табличные данные скорости КBM ${ }^{6}$.

\section{3 Основные результаты и выводы}

Сравнительный анализ показал, что существует сильная связь между $V_{C M E}$ и интегральным потоком микроволновых всплесков $\int F d t$ в диапазоне 2.7-15.4 ГГц. В частности, на рис. 1а показана соответствующая зависимость на частоте 8.8 ГГц, где $N$ - количество событий. Для подавляющего большинства протонных событий, обозначенных черными значками, коэффициент корреляции $r \approx 0.80$. На рис. 1а прямая линия соответствует линейной регрессии. Из 14 событий, обозначенных белыми значками, 7 были отождествлены авторами, а остальные 7 - взяты из каталога Международного цен-

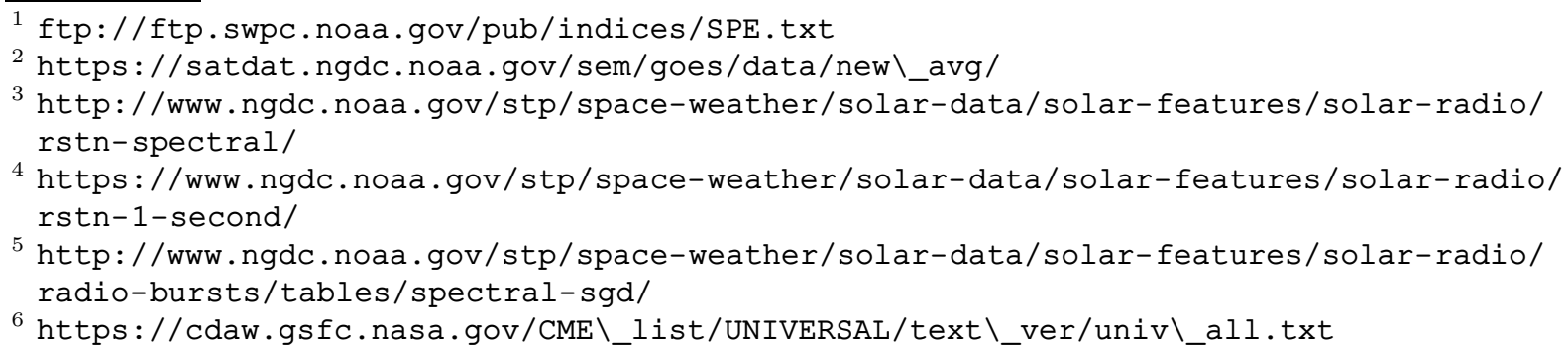




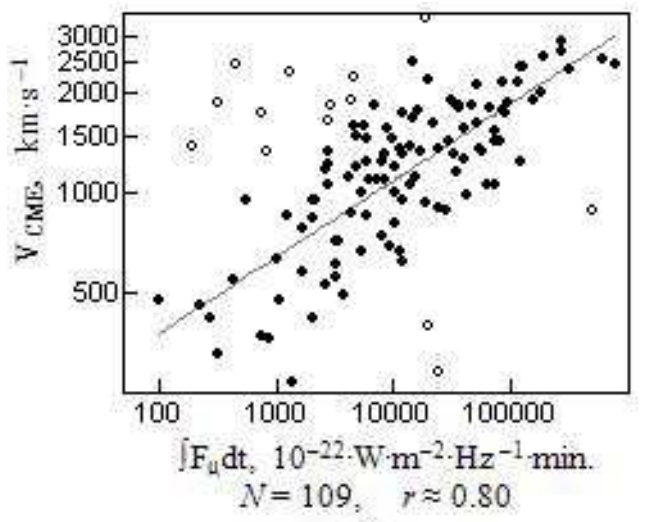

a).

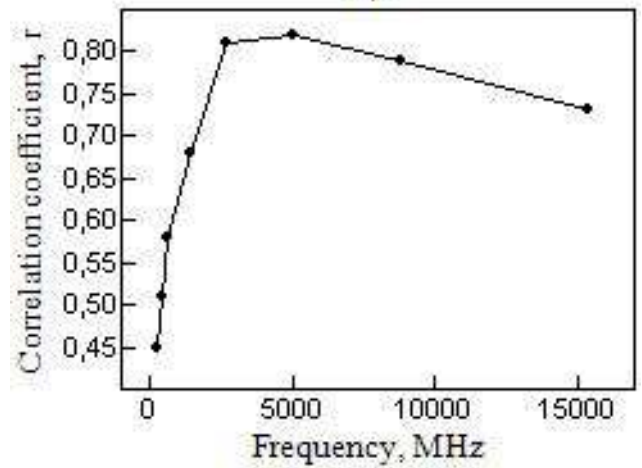

b).

Рис. 1. Связь скорости КВМ с интегральным потоком континуальных радиовсплесков (а) и зависимость значения коэффициента корреляции от частоты (b)

тра данных. На наш взгляд, их аномальное поведение в первую очередь обусловлено недостаточно точным отождествлением протонных вспышек с КВМ. Действительно, для серии накладывающихся протонных событий процедура отождествления может быть неоднозначной, поскольку в ней могут присутствовать как западные, так и восточные события, разнесенные по времени от нескольких часов до 3-х суток. На рис. $1 б$ обращает на себя также внимание, что связь между $V_{C M E}$ и интегральным потоком континуальных радиовсплесков $\int F d t$ значительно падает с уменьшением частоты, и уже в метровом диапазоне на частоте 245 МГц коэффициент корреляции не превышает 0.45.

Нами была также исследована связь уменьшения скорости частотного дрейфа всплесков II типа, генерируемых ударными волнами, с параметрами $\mu$-всплесков. Для анализа использовались оригинальные записи радиоизлучения Солнца на 8 фиксированных частотах в диапазоне 245-15400 МГц, полученные на RSTN. Сравнительный анализ показал, что существует достаточно убедительная связь уменьшения скорости частотного дрейфа всплесков II типа с характерным временем нарастания $\mu$ всплесков на частоте 8.8 ГГц. Коэффициент корреляции в этом случае достигает 0.66 .

Мы исследовали также зависимость скорости ударных волн от интегральных потоков $\int F_{\mu} d t$ для $\mu$-всплесков на частоте 8.8 МГц. Как оказалось, коэффициент корреляции составляет лишь около 0.55 , что с учетом полученных выше результатов свидетельствует в пользу импульсного происхождения ударных волн непосредственно в области вспышечного энерговыделения.

Работа выполнена при частичной поддержке Международного фонда технологий и инвестиций.

\section{Литература}

Дайбог (Daibog E.I. et al.) // Izvestiya AN SSSR. Seriya fizicheskaya. 1987. V. 51. P. 1825.

Дайбог (Daibog E.I. et al.) // Письма в Астрон. журн. 1989. V. 15. P. 991.

Очелков (Ochelkov Yu.P.) // Geomagn. Aeron. 1986. V. 26. P. 1007. 